\title{
Upper limb phantom pain - A case of difficult pain control
}

Maria Máximo ${ }^{\text {, João Borges }}{ }^{2}$, Célia Xavier $^{3}$, Paula Victor $^{3}$

${ }^{1}$ Anesthesiology Service, Hospital Prof. Doutor Fernando Fonseca E.P.E.

${ }^{2}$ Anesthesiology Service, Hospital do Divino Espírito Santo de Ponta Delgada E.P.E.

${ }^{3}$ Anesthesiology Service, Centro Hospitalar Lisboa Norte

\section{Introduction}

Phantom pain - present in up to $70 \%$ of amputees
Complex physiology and challenging treatment
One of the main causes

of acute pain

\section{Clinical case}

- 9,23 years old, $50 \mathrm{~kg}$, ASA III

- PH: Femoral osteossarcoma (2010); bone metastization (proximal humerus - ulceration with resistant $P$. aeruginosa infection)

- Medicated with SOS transdermal fentanyl $75 \mu \mathrm{g} / \mathrm{h}$

- She was proposed for a inter-thoraco-scapular amputation of the upper left limb

Intraoperative

\section{PACU}

Balanced general anesthesia

- Fentanyl $-3 \mu \mathrm{g} / \mathrm{kg}$ bolus

- Ketamine infusion- $4 \mu \mathrm{g} / \mathrm{kg} / \mathrm{min}$

- Direct brachial plexus infiltration (supraclavicular) - Ropivacaine 0,75\% 10 $\mathrm{mL}+$ Perineural catheter placement

- Paracetamol 1g, Metamizole 2g, Ketorolac $30 \mathrm{mg}$, Morphine $5 \mathrm{mg}$

- Paracetamol 1g 4id EV

- Metamizole 1g 3id EV

- Pregabaline $50 \mathrm{mg}$

- Ketamine infusion $4 \mu \mathrm{g} / \mathrm{kg} / \mathrm{min}$
On emergence:

- Phantom pain of the amputated limb $(10 / 10)$

- No relief after EV morphine and perineural ropivacaine $0,2 \%$ boluses

- Ropivacaine 0,2\% $5 \mathrm{~mL} / \mathrm{h}$ perineural infusion

- Morphine PCA $2 \mathrm{mg} / \mathrm{h}+1 \mathrm{mg}$ bolus

Discharge after 48 hours with partial pain control (7/10)

- Paracetamol 1g 4id EV

- Metamizole 1g 3id EV

- Pregabaline $250 \mathrm{mg} / \mathrm{day}$ Duloxetine $60 \mathrm{mg}$ id

- Ketamine infusion $9 \mu \mathrm{g} / \mathrm{kg} / \mathrm{min}$
- Ropivacaine 0,375\% $10 \mathrm{~mL}$ 6id

- Morphine PCA 2mg/h + bólus $1 \mathrm{mg}$

Slow titration; Improvement on PO D10, after initiating psychological support and hypnosis

\section{Discussion and Conclusion}

Phantom pain is multifactioral and has a complex treatment. Morphine, gabapentinoids, ketamine and regional analgesia have proven efficacy as part of multimodal

Pharmacological treatment may not be effective.

Prevention and treatment of post-amputation pain with specific and directed interventions should be a main concern of the anesthesiologist.

Perioperative psychological support, and alternative therapeutics should be considered as part of a multimodal treatment scheme. 